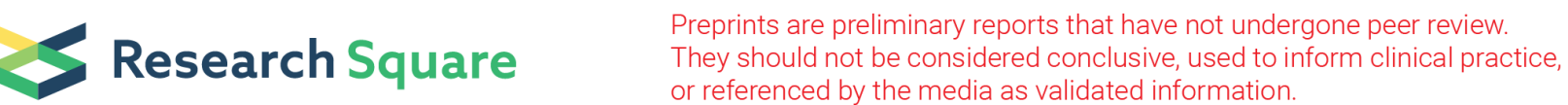

\section{The Inhibition of Autophagy Flux Mediated by Neferine Promoted the Apoptosis of HNSCC through the Accumulation of p62/SQSTM1}

Fengshuo Zhu

Shanghai Jiao Tong University School of Medicine

Xiaoguang Li

Shanghai Jiao Tong University School of Medicine

Xiao Tang

Shanghai Jiao Tong University School of Medicine

Junjian Jiang

Shanghai Jiao Tong University School of Medicine

Yu Han

Shanghai Jiao Tong University School of Medicine

Yinuo Li

Northwest University

Zhonglong Liu

Shanghai Jiao Tong University School of Medicine

Yue He ( $\nabla$ heyuejiuyuan@163.com )

Shanghai Jiao Tong University School of Medicine https://orcid.org/0000-0002-1015-4156

\section{Research}

Keywords: neferine, apoptosis, autophagy, head and neck squamous cell carcinoma

Posted Date: May 17th, 2020

DOI: https://doi.org/10.21203/rs.3.rs-28655/v1

License: (1) (1) This work is licensed under a Creative Commons Attribution 4.0 International License. Read Full License 


\section{Abstract}

Background: Head and neck squamous cell carcinoma (HNSCC) is one of the most common malignancies worldwide. Patients usually have very poor prognosis because of metastasis and chemoresistance. There is an imperative need to explore more effective chemotherapeutic agents. Neferine, a bisbenzylisoquinoline alkaloid isolated from the seed embryo of Lotus, exerts antitumor effects via regulating apoptosis and autophagy pathways, which becomes a potential therapeutic option for HNSCC. Methods: Cell viability and proliferation was determined by the CCK8 and colony formation assay. Cell cycle and apoptosis analysis were assessed by flow cytometry with Annexin V-FITC/PI staining. Intracellular ROS levels were determined by the DCFH-DA fluorescence. Autophagy flux was detected by transmission electron microscopy, transfected GFP-RFP-LC3 fluorescence and western blot for related markers in the presence or absence of neferine and chloroquine.

Results: Neferine inhibited the growth and induce the apoptosis of HNSCC cells both in vitro and vivo. Further analysis revealed that neferine activated ASK1/JNK pathway by increasing reactive oxygen species, then induced apoptosis of and regulated canonical autophagy in HNSCC cells. The study also revealed the novel pro-apoptosis mechanism through the activation of Caspase8 mediated by p62 due to the inhibition of autophagy flux.

Conclusions: These findings explore the anti-cancer effect of neferine, providing new insights into the crosstalk between apoptosis and autophagy mediated by p62 in HNSCC. The inhibition of autophagy flux by neferine might be a potential adjuvant therapy to HNSCC.

\section{Backgroud}

Head and neck squamous cell carcinoma (HNSCC) starts from the mucosal surfaces of oral cavity, oropharynx, larynx, and hypopharynx, accounting for more than $90 \%$ of the cancers of the head and neck[1]. As the sixth most common cancer by incidence worldwide, HNSCC is very aggressive and characterized by complex genetic alterations. Current treatment algorithms for HNSCC consist of surgical eradication, radiotherapy and chemotherapy. Even though there have been many advances in those modalities, the recurrence rates is still very high[2] owing to resistance to chemotherapy, and the overall survival of patients remains very low[3]. Therefore, there is a pressing need to explore novel chemotherapeutic agents for preventing cancer occurrence or delaying cancer progression.

Compounds derived from plants have made a significant contribution to the development of novel anticancer therapeutics. Nelumbo nucifera, commonly known as lotus, is widely used in Indian and Chinese medicine for cardiovascular[4] and pulmonary[5] diseases, as well as nervous disorders[6]. Neferine is the major bisbenzylisoquinoline alkaloid isolated from the seed embryo of Lotus. Recently, increasing data suggest neferine exert antitumor effects by various pathways. Some studies show that neferine inhibits proliferation and growth of choriocarcinoma[7], osteosarcoma[8], hepatocellular carcinoma[9] and lung cancer cells[10]. Neferine also could suppress migration of gastrointestinal 
stromal tumor cells[11] and glioma cells[12]. Recent researches suggest neferine inducing autophagy[13] and inhibiting the angiogenesis[14] of ovarian cancer cells. However, the effects of Neferine on HNSCC have never been elucidated.

(Macro) autophagy is a major intracellular catabolic mechanism that directs degration of cytoplasmic components and organelles in lysosome[15], which plays either a cytoprotective or cytopathic role in response to various stresses, such as therapeutic stress. Autophagic flux denotes the complete process of autophagy including autophagosome biogenesis, maturation, fusion with lysosomes, and breakdown of autophagic substrates inside the lysosome[16]. Induction or inhibition of the autophagy flux using compounds has shown promise in the treatment of diseases such as cancer[17]. As another critical catabolic pathway essential for response to toxic agents, apoptosis has an extensive crosstalk with autophagy[18]. And these two pathways are crucial for the development of effective cancer therapeutics.

We hypothesized that neferine is a promising alternative therapeutic agent for HNSCC. In this study, we determined whether neferine has anti-proliferative and anti-progression effects on HNSCC cell lines, particularly via the inhibition of autophagy flux and then promoted the neferine-induced apoptosis of HNSCC through the accumulation of P62/SQSTM1.

\section{Methods}

\section{Cell proliferation assay}

Cell viability was determined by the CCK8 assay kit (Dojindo). Briefly, the cells were seeded at a density of $5 \times 10^{3}$ cells per well of 96 -well plate, in $200 \mu$ l DMEM medium (10\% FBS). After incubation with different concentrations of neferine, $10 \mu \mathrm{l}$ of CCK-8 reagent was added to each well and the absorbance was measured at $450 \mathrm{~nm} 2 \mathrm{~h}$ later. All experiments were repeated three times.

\section{Clonogenic assay}

For the clonogenic assay, single-cell suspensions were prepared and 500 cells were seeded in 6-well plates overnight. Cells were treated with various concentrations of Neferine for 2 weeks. Cell colonies were fixed with Methanol, and stained by $0.1 \%$ crystal violet, then photographed and scored. Colonies of more than 50 cells were counted to determine the surviving fraction. All experiments were repeated three times.

\section{Cell cycle analysis}

For the cell cycle analysis, single-cell suspensions were prepared and $3 \times 10^{4}$ cells were seeded in 6 -well plates overnight. After treated with indicated concentrations of neferine for $24 \mathrm{~h}$, the cells were washed three times with PBS, trypsinized and collected by centrifugation at $1000 \mathrm{rpm}$ for $3 \mathrm{~min}$. Then, cells were fixed in $3 \mathrm{ml}$ of cold $75 \%$ ethanol at $-20^{\circ} \mathrm{C}$ overnight. After centrifugation at $1000 \mathrm{rpm}$ for $5 \mathrm{~min}$, cells were washed by $2 \mathrm{ml}$ cold PBS and centrifugated gain, cells were resuspended and incubated with $0.5 \mathrm{ml}$ 
of PBS containing $100 \mu \mathrm{g} / \mathrm{ml}$ RNase (Beyotime) and $5 \mu \mathrm{g} / \mathrm{ml}$ propidium iodide (Yeasen) at room temperature for $30 \mathrm{~min}$. Cell cycle distribution was analyzed by flow cytometry (BD Biosciences).

\section{Annexin V apoptosis assay}

Apoptotic cells were identified by FITC Annexin V Apoptosis Detection Kit (BD Bioscience). $3 \times 10^{4}$ cells were seeded in 6-well plates overnight. After treated with indicated concentrations of neferine for $48 \mathrm{~h}$, the cells were washed three times with PBS, trypsinized and collected. Cells were resuspended in binding buffer and incubated at room temperature for $15 \mathrm{~min}$ in the dark after $5 \mu \mathrm{FITC}$ Annexin V and $10 \mu \mathrm{II}$ were added. Apoptotic cells were analyzed by flow cytometry in $1 \mathrm{~h}$.

\section{ROS assay}

Intracellular ROS levels were determined by the DCFH-DA fluorescent probe (Beyotime). $3 \times 10^{4}$ cells were seeded in 6-well plates overnight. After treated with indicated concentrations of neferine for $12 \mathrm{~h}$, the cells were incubated with DCFH-DA for 30 min and washed three times with PBS. Then DCF fluorescence was detected by flow cytometry (FITC-Channel). Fluorescence microscopy (Carl Zeiss) was used to photograph the cell.

\section{Cell migration assay}

The cell migration assay was performed using the Transwell system (24-wells, 8- $\mu \mathrm{m}$ pore size with polycarbonate membrane, Corning Costar). $5.0 \times 10^{5}$ Cells were suspended in serum-free medium and added to the upper chamber, $700 \mu \mathrm{L}$ of complete medium (10\% FBS) was added to the lower chamber. After $4 \mathrm{~h}$, the media in the upper chambers were replaced by media with various concentration of neferine. After $36 \mathrm{~h}$, the chambers were fixed with paraformaldehyde, and stained by $0.1 \%$ crystal violet. The non-migrated cells were wiped off from upper surface of chamber using cotton swabs. The successfully migrated cells were last counted on 3 random fields.

\section{Scratch assay}

Scratch assays were applied to determine cell mobility affected by Neferine. $1 \times 10^{6}$ cells were seeded on a 6-well plate in the complete medium overnight to obtain a full confluent monolayer. After $12 \mathrm{~h}$ of starvation, a $20 \mu \mathrm{L}$ pipette tip was used to make a straight cell-free "scratch" on the well plate. The well was washed with PBS and added serum-free medium with different concentration of Neferine. Last, the migration of cells was photographed using a phase contrast microscope. Photographs were taken of cells at the matching reference points initially and then at $12 \mathrm{~h}$ intervals. The decreased square of the scratch were analyzed quantitatively.

\section{Western blot analysis}

Briefly, cells were collected after specific treatments and then lysed with RIPA. The concentration of protein was assayed using the Pierce BCA Protein Assay Kit (Thermo). 20 $\mu$ g of each sample was resolved 
into SDS-PAGE gels and then electro-transferred onto a PVDF membrane (Millipore). The membrane was blocked with $5 \%$ skimmed milk for $1 \mathrm{~h}$ at room temperature and incubated overnight with primary antibody at $4{ }^{\circ} \mathrm{C}$. The membrane was washed and incubated with HRP conjugated secondary antibody for $1 \mathrm{~h}$. The protein bands were detected by a chemiluminescence system (Immobilion Western, Millipore). $\beta$-actin was used as an internal control.

The related antibodies $₫$ Anti-cleaved caspase 3 (CST and Santa); Anti-cleaved caspase 9, Anti-cleaved PARP1, anti-Beclin 1 and Anti-p62 (Proteintech); Anti-Bax (Beyotime) and Anti-Bcl2 (Santa); Anti-LC3-I/II (Abcam). The secondary mouse and goat antibodies (Beyotime).

\section{Transmission Electron Microscopy (TEM) assay}

After treated with neferine for $24 \mathrm{~h}, \mathrm{HN} 30$ and CAL27 cells were fixed with glutaraldehyde and $\mathrm{O}_{5} \mathrm{O}_{4}$, dehydrated with graded ethanol, embedded in propylene oxide, sectioned at 70nm and stained with uranyl acetate nd lead citrate. The autophagosomes in cells are visualized with the electron microscope as described in previous method[19,20].

\section{Fluorescence image}

Cells were seeded in 6-well plates overnight and then transfected GFP-RFP-LC3 adenovirus (Beyotime), after $24 \mathrm{~h}$, the medium was removed and replaced with various concentrations of Neferine for another 24 h. Autophagic flux was measured under the fluorescence microscopy (Carl Zeiss).

\section{RNA interference}

The sequence of siRNAs against p62 was 5'-GCATTGAAGTTGATATCGAT-3, as previously published[21]. Cells were seeded in six-well plate and transfected with siRNA using Lipofectamine3000 (Invitrogen). After $72 \mathrm{~h}$, the transfected cells in parallel conditions were used for neferine experiments.

\section{Xenograft mice model}

The animal experiment was performed according to the Ethics Review Board at the Shanghai Ninth People Hospital. The CAL27 ( $2 \times 10^{6}$ cells) suspended in 20\% Matrigel (BD Biosciences) were injected to BALB/c nude mice (CRL, China) subcutaneously. The mice were treated with neferine $(10 \mathrm{mg} / \mathrm{kg})$ and PBS (control) by intraperitoneal administration after a week. The volumes of tumor were measured every four days. The mice were euthanatized and tumor were required at end of the experiment.

\section{Immunohistochemistry staining}

The slides were disposed through deparaffinage, rehydration, antigen repairation, the endogenous peroxidase inactivation and nonspecific antigen blocking. The slides were incubated with primary antibody overnight, the secondary antibody $15 \mathrm{~min}$. Staining was displayed by a DAB reagent (Zhongshan). The intensity and proportion of staining were scored by 2 pathologists. 


\section{Statistical analysis}

SPSS version 18.0 software was used for statistical analysis. For each of the 3 independent experiments, data are presented as the mean \pm SEM. Statistical comparisons between 2 samples were performed using the Student's t-test and multiple comparison were analyzed by one-way ANOVA test. Differences were considered statistically significant when $\mathrm{P}<0.05$.

\section{Results}

\section{Neferine exerted the anti-cancer effect and induced the apoptosis in HNSCC cells.}

Three human head and neck squamous cell carcinoma cell lines (HN6, HN30 and CAL27) were used to detect the inhibitory effect of neferine on cell proliferation by CCK8 assay. As shown in Fig. 1A, cell viabilities of HN6, HN30 and CAL27 were significantly decreased in a dose- and time-dependent manner with the treatment of neferine. The IC50 values at $72 \mathrm{~h}$ were $13.13 \mu \mathrm{M}$ in HN6, $21.74 \mu \mathrm{M}$ in HN30, $22.54 \mu \mathrm{M}$ in CAL27 (Fig. 1A). Similarly, colony formation was increasingly inhibited as the neferine concentration increased in HN6, HN30 and CAL27 cells (Fig. 1B). Hence, the cell cycle distribution of cells treated with various concentrations of neferine for $24 \mathrm{~h}$ was examined by flow cytometry. Neferine caused an obvious $\mathrm{G} 1$ cell cycle arrest in HN6, HN30 and CAL27 cells. The percentage of cells at G1 phase was increased to $63.78 \%(20 \mu \mathrm{M})$ from $44.86 \%(0 \mu \mathrm{M})$, while the percentages of cells at $S$ phase were decreased to $22.14 \%(20 \mu \mathrm{M})$ from $41.42 \%(0 \mu \mathrm{M})$ in the HN6 cells. The similar results also could be observed in another two cell lines (Fig. 1C and Fig. S1A). All of these results indicated neferine exerted its inhibitory effect through inducing cell cycle arrest in HN6, HN30 and CAL27 cells. To investigate the effects of Neferine on HNSCC cells motility, the scratch healing assay and transwell assay were performed. Neferine suppressed the healing and the migration of HNSCC cells in dose-dependent manner (Fig. S1B and S1C). Overall, these results indicated that neferine exihibited the potential anti-cancer property.

Next, we investigated whether neferine exerted its anti-cancer effect through inducing cell apoptosis in HN6, HN30 and CAL27 cells. HNSCC cells were treated with 0-20 $\mu \mathrm{M}$ neferine for $48 \mathrm{~h}$, then apoptotic cells were determined by Annexin-V staining. Neferine dose-dependently increased the percentages of apoptotic cells from $7.51 \%(0 \mu \mathrm{M})$ to $11.81 \%(5 \mu \mathrm{M}), 27.90 \%(10 \mu \mathrm{M}), 49.10 \%(15 \mu \mathrm{M})$ and $64.70 \%$ $(20 \mu \mathrm{M})$ in HN6 cells. Similar tendency could be observed in CAL27 and HN30 cells (Fig. 1D and Fig. S2A). Then apoptosis-related proteins, including Bcl-2, Bax, cleaved caspase-8, caspase9, caspase-3 and PARP-1 were examined in HN30 and CAL27 cells. The treatment of neferine obviously decreased the ratio of Bcl-2/Bax, and triggered the cleavage of caspase8, caspase9, caspase3 and PARP-1 in a dose- and time- dependent manner (Fig. 1E).

\section{Neferine promoted the generation of autophagosomes but inhibited the autophagy influx.}

Given the indistinct reciprocal interaction between apoptosis and autophagy induced by neferine, we also explored the effect of neferine on autophagy in HNSCC cell lines. The expression of Beclin1 was analysed 
for its important role in the initial steps of canonical autophagy. Beclin1 in the HN30 and CAL27 cells was increased after treatment with neferine in a dose- and time-dependent manner (Fig. 2A). LC3 and p62 were universal markers in autophagy assessment, for LC3-I would convert to LC3-II and LC3-II would increase as the autophagosome matured; p62 would be degraded in the autolysosome and the level of p62 would decrease as the autophagy flux activated. In our experiment, dose- and time-dependent increase in the conversion of LC3-I to LC3-II isoform was observed in neferine-treated HN30 and CAL27 cells (Fig. 2A), which indicated autophagosome matured. However, the protein level of p62 was also increased. Transmission electron microscopy (TEM) showed neferine treatment increased the amounts of vacuoles containing cytoplasmic materials and part of them were multivesicular bodies (MVBs), a characteristic feature of degradative autophagic vacuoles (Fig. 2B). To further explore the influence of neferine on autophagy, the autophagy flux was also monitored using an autophagosome-lysosome fusion inhibitor, chloroquine[22]. We examined the effect of neferine and chloroquine co-treatment on LC3 levels. LC3-II level in chloroquine group was less than that in the chloroquine + neferine group, which indicated the neferine promoted the autophagosome biosynthesis. The p62 level wasn't further increased in the presence of chloroquine, suggesting that neferine-induced upregulation of p62 might be due to the inhibition of autophagic degradation (Fig. 2C), The difference of LC3-II levels between chloroquine + neferine and neferine group was less than the difference between chloroquine + neferine and chloroquine group. This also revealed that neferine could inhibit the autophagosome degradation. To further confirm these results, we also examined the autophagy flux through double-labelling fluorescent LC3 adenovirus (mCherry-GFP-LC3[23, 24] (Fig. 2D and 2E). Colocalization (yellow) of both GFP (green) and mCherry (red) fluorescence implied the generation of autophagosome. In contrast, a mCherry signal without GFP referred to an autolysosome which indicated the fluent autophagic flux. The quantification of red (mCherry + GFP-) and yellow (mCherry + GFP+) puncta per cell indicated that neferine increased biosynthesis but inhibited the degradation of autophagosome (red and yellow puncta). All the results indicated that neferine induced the autophagosome biosynthesis but inhibited the autophagy flux at final degration step in HNSCC cell lines.

\section{Neferine induced the ROS hypergeneration and activated the ASK1/JNK pathway to promote the apoptosis and autophagosome biosynthesis in HNSCC cells.}

ROS was a vital inducer of apoptosis and autophagy $[25,26]$. Therefore, the amount of ROS in HNSCC cells treated with neferine for 24 hours was measured using the oxidation-sensitive fluorescent probe dichlorofluorescein (DCF) by flow cytometry. The mean intensity of DCF fluorescence in neferine-treated HN30 cells were increased by $2.62(10 \mu \mathrm{M})$ and $3.01(20 \mu \mathrm{M})$ folds compared to that in control cells $(0 \mu \mathrm{M})$; Similar results could be observed in CAL27 cells (Fig. 3A). The fluorescence images also confirmed the increase of ROS (Fig. 3B). It's reported that ROS could activate the ASK1/JNK signal pathway to induce mitochondrial apoptosis by caspase family and Beclin1-dependent autophagy[27, 28]. Hence, we dectected the ASK1/JNK pathway in the cells treated with neferine and found neferine upregulated the phosphorylation of ASK1/JNK in dose- and time-dependent way but didn't alter the overall ASK1/JNK expression (Fig. 3C). 
Neferine-induced apoptosis was partially mediated by the suppression of autophagy influx in HNSCC cells.

The HN30 and CAL27 cells were treated with neferine $(20 \mu \mathrm{M})$ in the presence or absence of chloroquine $(10 \mu \mathrm{M})$ for 48 hours. The inhibition of autophagic flux by chloroquine enhances the inhibitory effect of neferine on cell viability (Fig. 4A). Then we measured the apoptosis by flow cytometry and detected the expression of cleaved caspases and PARP1. Chloroquine-mediated autophagy flux inhibition moderately intensified neferine-induced apoptosis, which was consistent with the activation of caspase-8, caspase-3 and PARP1(Fig. 4B, 4C and Fig. S3). The results indicated the block of autophagy flux by neferine promoted apoptosis of HN30 and CAL27 cells. However, the expression of cleaved caspase-9 showed no obvious change (Fig. 4C). The results indicated that the pro-apoptotic enhancement by chloroquineinduced autophagic influx inhibition was executed by caspase-8, but not caspase- 9 .

The accumulation of p62 due to neferine-induced autophagy influx inhibition promoted the apoptosis in HNSCC cells.

Previous researches revealed that p62 acted as a "bridge" between apoptosis and autophagy[29, 30]. The autophagy flux inhibition by neferine elevated the level of $p 62$, so we next investigated the mechanism of p62-mediated pro-apoptosis through autophagy flux inhibition by neferine. The flow cytometry analysis revealed that the knockdown of p62 alleviated the neferine-induced apoptosis (from 43.3-29.7\%) in CAL27 cells (Fig. 4D). The apoptosis related protein (cleaved caspase-8, caspase-3 and PARP1) are also decreased, however the change of cleaved caspase-9 was not obvious (Fig. 4E). The results manifested that the accumulation of p62 induced by neferine activated the caspase- 8 to promote the apoptosis of HNSCC.

\section{Neferine inhibited the HNSCC carcinoma growth by induction of apoptosis and autophagy in vivo.}

Above studies had shown that neferine inhibited the growth of HNSCC cells in vitro. Next, xenografts using CAL27 cells were constructed to explore the effect of neferine on HNSCC in vivo. The volumes of tumors treated with neferine were significantly smaller than the control. The growth rate of tumors treated with neferine was also slower than the control (Fig. 5A and 5B). Furthermore, the Immunohistochemical staining of xenograft tumor tissues (Fig. 5C) showed that treatment with neferine reduced the expression of Ki67, which was a marker of cell proliferation. In the meanwhile, neferine elevated the expression of cleaved Caspase-3 and cleaved PARP1 in the nucleus, which indicated the neferine induced the apoptosis of xenograft tumors. In accordance with the in vitro results, the neferine also increased the level of LC3-II and p62, which implied the neferine induced the autophagosome generation and inhibited the autophagy influx.

\section{Discussion}

Neferine was an attractive reagent that was more toxic to cancer cells than non-transformed cells[13]. The induction of cell apoptosis and autophagy has been reported in the previous research[14, 31], 
however a conflicting study covered that neferine was an autophagy inhibitor[32]. Besides, the reciprocal interaction between apoptosis and autophagy induced by neferine was indistinct. Hence, this study aimed to elucidate the influence of neferine on autophagy and apoptosis in HNSCC.

To our knowledge, this was the first study about the effects and mechanism of neferine in HNSCC. We introduced a multidimensional evaluation in this study and found the pharmacological effect of neferine was undoubtedly versatile. Firstly, we discovered that neferine inhibited the viability and colony formation of cancer cells and could induce the arrest of G1/S phase in HNSCC, which could also be observed in ovarian carcinoma by down-regulating expression of cyclin E1[14]. Neferine suppressed the healing and the migration of HNSCC cells, which also indicated the potential anti-cancer property of neferine. The anti-cancer effect of neferine was also proved in vivo. In the further study, we found that neferine induced apoptosis and autophagy in company with the increase of ROS level and the activation of ASK1/JNK pathway. The ROS was an initiator to activate ASK1 (oxidative stress sensor) and JNK[33, 34]. The mitochondrial apoptosis induced by Bcl-2 inhibition through JNK and the canonical autophagy induced by JNK/Beclin-1 were sequential occurrences. Hence, neferine-induced ROS by was an executor signal for apoptosis and autophagy in the upstream. The results indicated that neferine-induced autophagosome biogenesis was determined by the Beclin1-dependent canonical autophagy pathway.

Autophagy was a crucial mechanism to degrade harmful cellular components to mediate metabolic adaptation and maintain energy homeostasis[35]. Several studies had revealed that autophagy exhibited paradoxical role, having either tumor-promotion or tumor-suppression effects on carcinoma[36, 37]. In the meanwhile, the inhibition of autophagy was regarded as a universal target in anti-cancer therapy[38]. In our research, neferine was a double-edged sword for autophagy in HNSCC. We found neferine induced autophagosome generation but inhibited the autophagy flux at the autophagosome degradation step with the conversion from LC3-I to LC3-II and accumulation of p62.

p62/SQSTM1 was involved in the formation of autophagosome and was degraded in the lysosomal proteases in accompany with autophagosome[39], as a result, the inhibition of autophagy flux caused the accumulation of p62. p62 was a scaffold protein that contained five domains that organize signaling traffic at critical decision points that controlled cell death and survival[40, 41]. The UBA domain of p62 interacted with ubiquitinated proteins, and p62 could recruite and oligomerize important signaling molecules[41]. It was reported that p62 provided a signal organizing interface to recruit poly-ubiquitinated caspase-8 and subsequently activated the caspase8[30]. Our research revealed that neferine induced the accumulation of p62, which would aggravate the apoptosis induced by neferine. This was also confirmed by the knockdown of p62 and all the results indicated that p62 might act as a bridge between autophagy and apoptosis induced by neferine.

When treated with reagents that induced proteasome inhibition and ER stress, cells could activate apoptosis directly through the caspase8[42]. This mechanism was strengthened by the function of LC-3 and p62[43,44]. We proposed a novel mechanism of neferine that the accumulation of p62 by autophagy influx inhibition promoted the apoptosis through activation of caspase8 independent of ROS in HNSCC. 
The involvement of p62 in neferine-induced cell apoptosis through caspase-8 activation broadened our horizon of mechanism for neferine-induced cell death. The crosstalk between autophagy and apoptosis occurred with $\mathrm{p} 62$ as a possible conjunction point in neferine-induced cell death. The activation of caspase8 synergized with the accumulation of p62 and it was plausible that the caspase 8 might interact with p62 at the autophagosome surface by neferine treatment.

\section{Conclusion}

Taking our findings of increasing apoptosis and interrupted autophagy flux, our study clarified the underlying pro-apoptosis mechanism of neferine in HNSCC through the inhibition of autophagy influx mediated by $\mathrm{p} 62$, providing new insights into the crosstalk between apoptosis and autophagy and also highlighted neferine as a potential agent to improve prognosis of HNSCC patients (Fig. 6).

\section{Declarations}

\section{Acknowledgments}

None.

\section{Authors' contributions}

ZFS, LXG, TX, HY and JJJ performed research and analyzed results; ZFS, LYN and LZL developed methodology and discussed results; ZFS, LYN and HY designed research, wrote the paper and supervised the study. All authors read and approved the final manuscript.

\section{Funding}

This work was supported by the National Natural Science Foundation of China [81570949].

\section{Ethics approval and consent to participate}

Yes.

\section{Consent for publication}

Yes. 


\section{Competing interests}

The authors declare that they have no competing interests.

\section{References}

1. Vigneswaran $\mathrm{N}$, Williams MD. Epidemiologic trends in head and neck cancer and aids in diagnosis. Oral Maxillofac Surg Clin North Am. 2014;26(2):123-41.

2. Yamano $Y$, et al. Identification of cisplatin-resistance related genes in head and neck squamous cell carcinoma. Int J Cancer. 2010;126(2):437-49.

3. Mortality GBD. and C. Causes of Death, Global, regional, and national life expectancy, all-cause mortality, and cause-specific mortality for 249 causes of death, 1980-2015: a systematic analysis for the Global Burden of Disease Study 2015. Lancet. 2016;388(10053):1459-544.

4. Qian JQ. Cardiovascular pharmacological effects of bisbenzylisoquinoline alkaloid derivatives. Acta Pharmacol Sin. 2002;23(12):1086-92.

5. Zhao L, et al. Neferine, a bisbenzylisoquinline alkaloid attenuates bleomycin-induced pulmonary fibrosis. Eur J Pharmacol. 2010;627(1-3):304-12.

6. Sugimoto $Y$, et al. Effects of extracts and neferine from the embryo of Nelumbo nucifera seeds on the central nervous system. Phytomedicine. 2008;15(12):1117-24.

7. Wang $G$, et al. Neferine hinders choriocarcinoma cell proliferation, migration and invasion through repression of long noncoding RNA-CHRF. Artif Cells Nanomed Biotechnol. 2019;47(1):4089-96.

8. Zhang $X$, et al. Neferine, an alkaloid ingredient in lotus seed embryo, inhibits proliferation of human osteosarcoma cells by promoting p38 MAPK-mediated p21 stabilization. Eur J Pharmacol. 2012;677(1-3):47-54.

9. Deng G, et al. The anti-tumor activities of Neferine on cell invasion and oxaliplatin sensitivity regulated by EMT via Snail signaling in hepatocellular carcinoma. Sci Rep. 2017;7:41616.

10. Poornima P, Weng CF, Padma VV. Neferine, an alkaloid from lotus seed embryo, inhibits human lung cancer cell growth by MAPK activation and cell cycle arrest. Biofactors. 2014;40(1):121-31.

11. Xue F, et al. Neferine inhibits growth and migration of gastrointestinal stromal tumor cell line GIST-T1 by up-regulation of miR-449a. Biomed Pharmacother. 2019;109:1951-9.

12. Liang HX, Sun LB, Liu NJ. Neferine inhibits proliferation, migration and invasion of U251 glioma cells by down-regulation of miR-10b. Biomed Pharmacother. 2019;109:1032-40.

13. Xu L, et al. Neferine induces autophagy of human ovarian cancer cells via p38 MAPK/ JNK activation. Tumour Biol. 2016;37(7):8721-9.

14. Zhang Q, et al. Anti-angiogenesis effect of Neferine via regulating autophagy and polarization of tumor-associated macrophages in high-grade serous ovarian carcinoma. Cancer Lett. 2018;432:144-55. 
15. Vakifahmetoglu-Norberg H, Xia HG, Yuan J. Pharmacologic agents targeting autophagy. J Clin Invest. 2015;125(1):5-13.

16. Mizushima N, Yoshimori T, Levine B. Methods in mammalian autophagy research. Cell. 2010;140(3):313-26.

17. Cheng $Y$, et al. Therapeutic targeting of autophagy in disease: biology and pharmacology. Pharmacol Rev. 2013;65(4):1162-97.

18. Su M, Mei Y, Sinha S, Role of the Crosstalk between Autophagy and Apoptosis in Cancer. J Oncol, 2013. 2013: p. 102735.

19. Lucocq JM, Hacker C. Cutting a fine figure: On the use of thin sections in electron microscopy to quantify autophagy. Autophagy. 2013;9(9):1443-8.

20. Eskelinen EL, et al. Seeing is believing: the impact of electron microscopy on autophagy research. Autophagy. 2011;7(9):935-56.

21. Pankiv S, et al. Nucleocytoplasmic shuttling of p62/SQSTM1 and its role in recruitment of nuclear polyubiquitinated proteins to promyelocytic leukemia bodies. J Biol Chem. 2010;285(8):5941-53.

22. Klionsky DJ, et al., Guidelines for the use and interpretation of assays for monitoring autophagy (3rd edition). Autophagy, 2016. 12(1): p. 1-222.

23. Kimura S, Noda T, Yoshimori T. Dissection of the autophagosome maturation process by a novel reporter protein, tandem fluorescent-tagged LC3. Autophagy. 2007;3(5):452-60.

24. Pankiv S, et al. p62/SQSTM1 binds directly to Atg8/LC3 to facilitate degradation of ubiquitinated protein aggregates by autophagy. J Biol Chem. 2007;282(33):24131-45.

25. Martindale JL, Holbrook NJ. Cellular response to oxidative stress: signaling for suicide and survival. J Cell Physiol. 2002;192(1):1-15.

26. Chen $Y$, Gibson SB. Is mitochondrial generation of reactive oxygen species a trigger for autophagy? Autophagy. 2008;4(2):246-8.

27. Liu J, Lin A. Role of JNK activation in apoptosis: a double-edged sword. Cell Res. 2005;15(1):36-42.

28. Tang D, et al. High-mobility group box 1 , oxidative stress, and disease. Antioxid Redox Signal. 2011;14(7):1315-35.

29. Philip NH, et al. Activity of Uncleaved Caspase-8 Controls Anti-bacterial Immune Defense and TLRInduced Cytokine Production Independent of Cell Death. PLoS Pathog. 2016;12(10):e1005910.

30. Zhang YB, et al. Autophagy protein p62/SQSTM1 is involved in HAMLET-induced cell death by modulating apotosis in U87MG cells. Cell Death Dis. 2013;4:e550.

31. Poornima P, Quency RS, Padma VV. Neferine induces reactive oxygen species mediated intrinsic pathway of apoptosis in HepG2 cells. Food Chem. 2013;136(2):659-67.

32. Xu T, et al. Neferine, is not inducer but blocker for macroautophagic flux targeting on lysosome malfunction. Biochem Biophys Res Commun. 2018;495(1):1516-21.

33. Zhao Q, et al. Pristimerin induces apoptosis and autophagy via activation of ROS/ASK1/JNK pathway in human breast cancer in vitro and in vivo. Cell Death Discov. 2019;5:125. 
34. Ma L, et al. Low glucose and metformin-induced apoptosis of human ovarian cancer cells is connected to ASK1 via mitochondrial and endoplasmic reticulum stress-associated pathways. J Exp Clin Cancer Res. 2019;38(1):77.

35. Li T, et al., Salinomycin induces cell death with autophagy through activation of endoplasmic reticulum stress in human cancer cells. Autophagy, 2013. 9(7): p. 1057-68.

36. Rosenfeldt MT, Ryan KM. The role of autophagy in tumour development and cancer therapy. Expert Rev Mol Med. 2009;11:e36.

37. Kondo Y, Kondo S. Autophagy and cancer therapy. Autophagy. 2006;2(2):85-90.

38. Sui $X$, et al. Autophagy and chemotherapy resistance: a promising therapeutic target for cancer treatment. Cell Death Dis. 2013;4:e838.

39. Ichimura Y, Komatsu M. Selective degradation of p62 by autophagy. Semin Immunopathol. 2010;32(4):431-6.

40. Moscat J, Diaz-Meco MT, Wooten MW. Signal integration and diversification through the p62 scaffold protein. Trends Biochem Sci. 2007;32(2):95-100.

41. Moscat J, Diaz-Meco MT. p62 at the crossroads of autophagy, apoptosis, and cancer. Cell. 2009;137(6):1001-4.

42. Hou W, et al. Autophagic degradation of active caspase-8: a crosstalk mechanism between autophagy and apoptosis. Autophagy. 2010;6(7):891-900.

43. Young MM, et al. Autophagosomal membrane serves as platform for intracellular death-inducing signaling complex (iDISC)-mediated caspase-8 activation and apoptosis. J Biol Chem. 2012;287(15):12455-68.

44. Pan JA, et al. Hyperactivation of the mammalian degenerin MDEG promotes caspase-8 activation and apoptosis. J Biol Chem. 2013;288(5):2952-63.

\section{Figures}




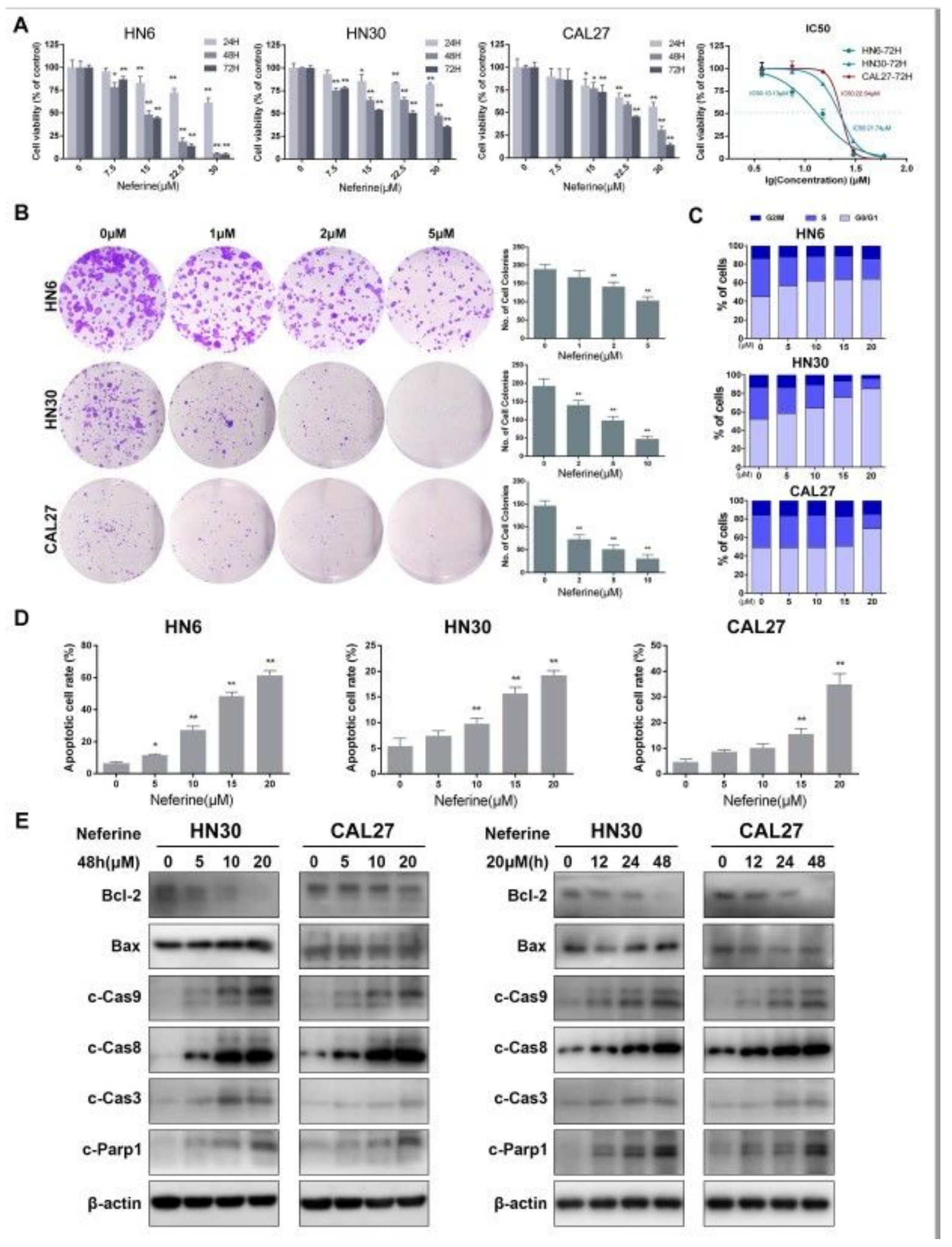

\section{Figure 1}

Neferine inhibited the cell viability and induced apoptosis in HNSCC cells. (A) Histograms indicated cell viabilities of HN6, HN30 and CAL27 with the treatment of neferine. The line chart shows the IC50 values at $72 \mathrm{~h}$ were $13.13 \mu \mathrm{M}$ in HN6, 21.74 $\mu \mathrm{M}$ in HN30, 22.54 $\mu \mathrm{M}$ in CAL27. (B) Plate colony formation assay showed an inhibitory effect of Neferine on HNSCC cells with indicated concentrations. (C) The cell cycle distribution of cells treated with various concentrations of neferine for $24 \mathrm{~h}$ was examined by flow 
cytometry. (D) Flow cytometric analysis of early and late apoptosis in treated HN6, HN30 and CAL37 cells with neferine. (E) Apoptosis-related proteins expression in HN30 and CAL37 cells treated with neferine was confirmed by western blot. Left panel, HN30 and CAL37 cells were treated with indicated concentrations of neferine for $48 \mathrm{~h}$. Right panel, HN30 and CAL37 cells were treated with neferine $(20 \mu \mathrm{M})$ for indicated time.

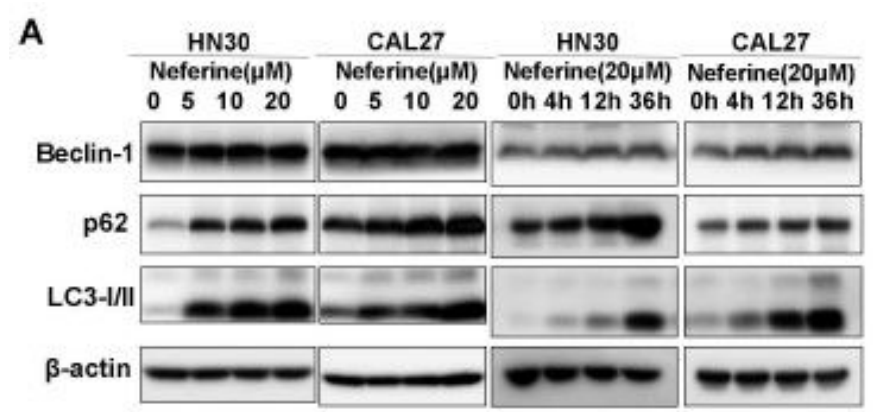

B
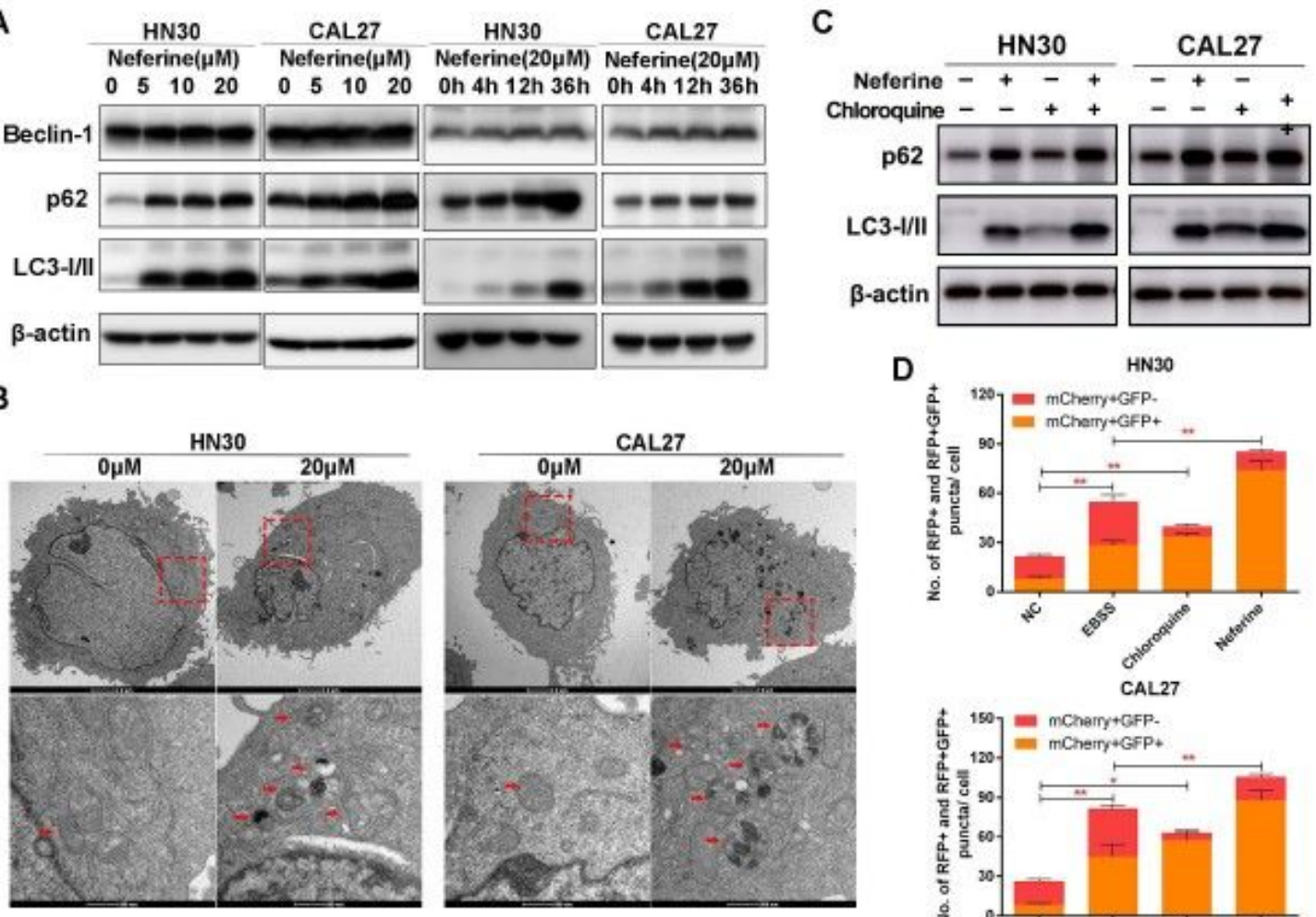

D

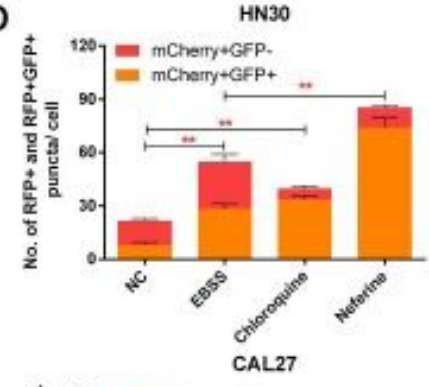

E
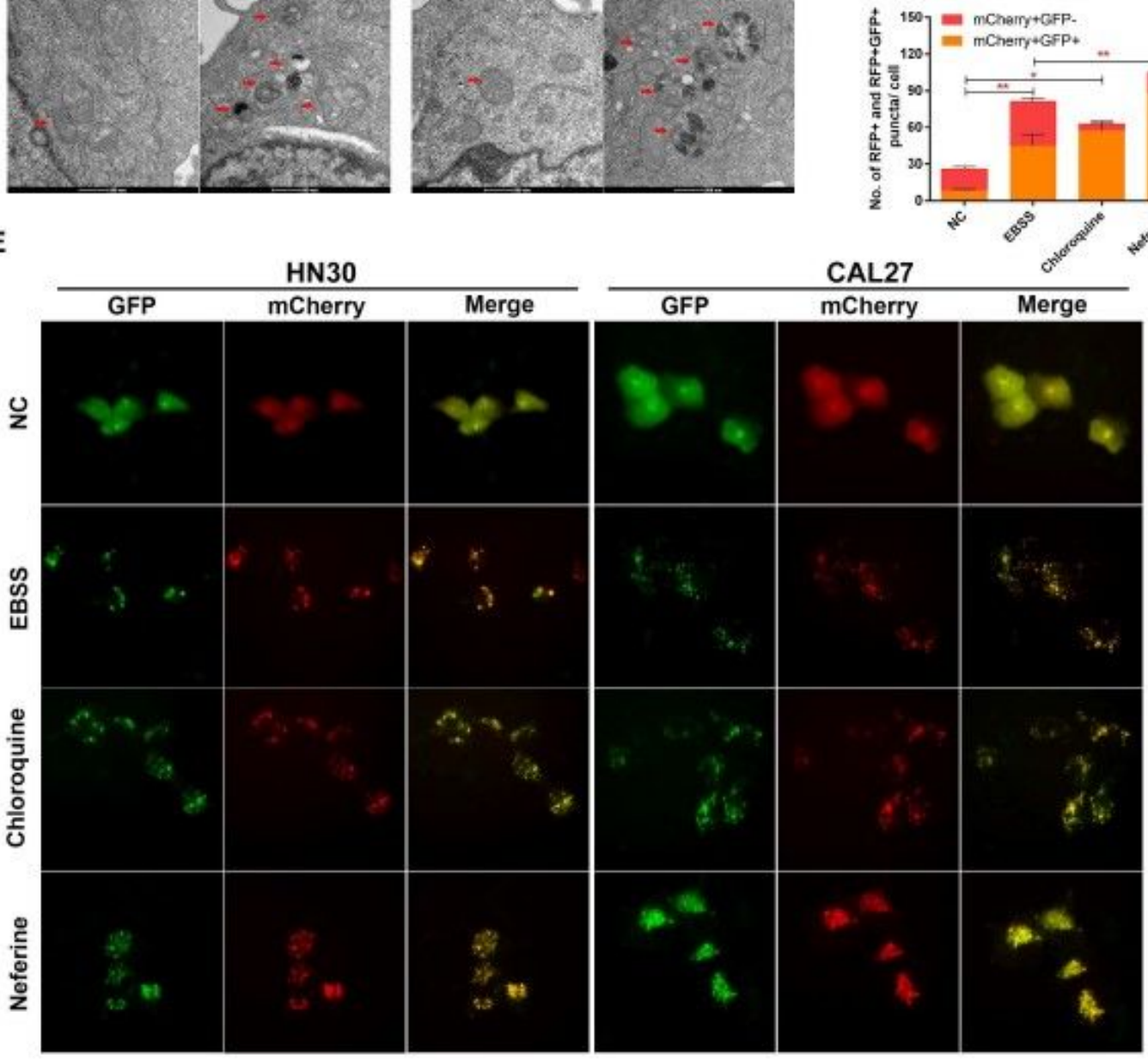

Figure 2 
Neferine promoted the generation of autophagosomes but inhibited the autophagic influx. (A) The expression of Beclin 1, p62 and LC3 was analysed by western blot in HN30 and CAL27 cells with the treatment of neferine with indicated concentrations or for indicated times. (B) Transmission electron microscopy (TEM) showed neferine treatment increased the amounts of vacuoles containing cytoplasmic materials and part of them were multivesicular bodies (MVBs), a characteristic feature of degradative autophagic vacuoles. (C) Western blot of p62 and LC3 expression in the HN30 and CAL27 cells with the neferine and chloroquine co-treatment. ( $\mathrm{D}$ and $\mathrm{E}$ ) Autophagy flux was examined through double-labelling fluorescent LC3 adenovirus (mCherry-GFP-LC3). Colocalization (yellow) of both GFP (green) and mCherry (red) fluorescence implied the generation of autophagosome. 
A
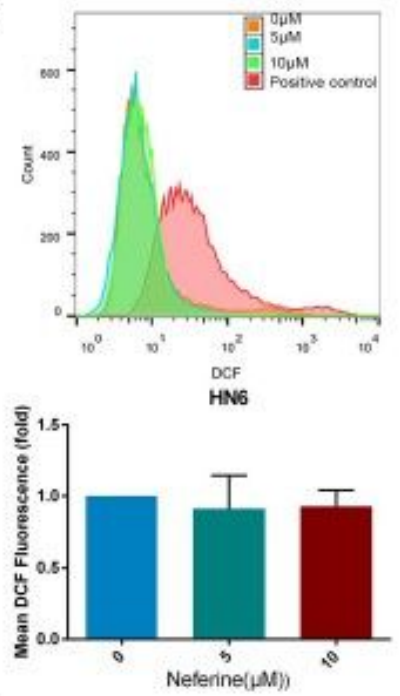

B
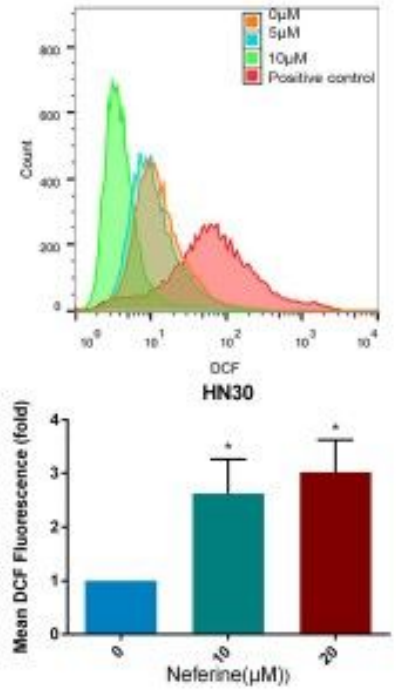

$10 \mu \mathrm{M}$
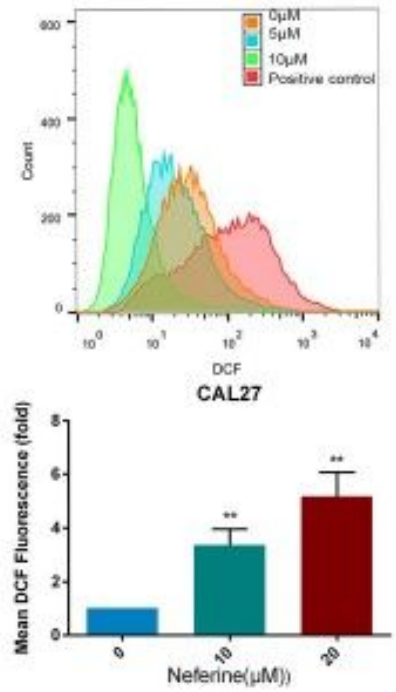

$20 \mu \mathrm{M}$

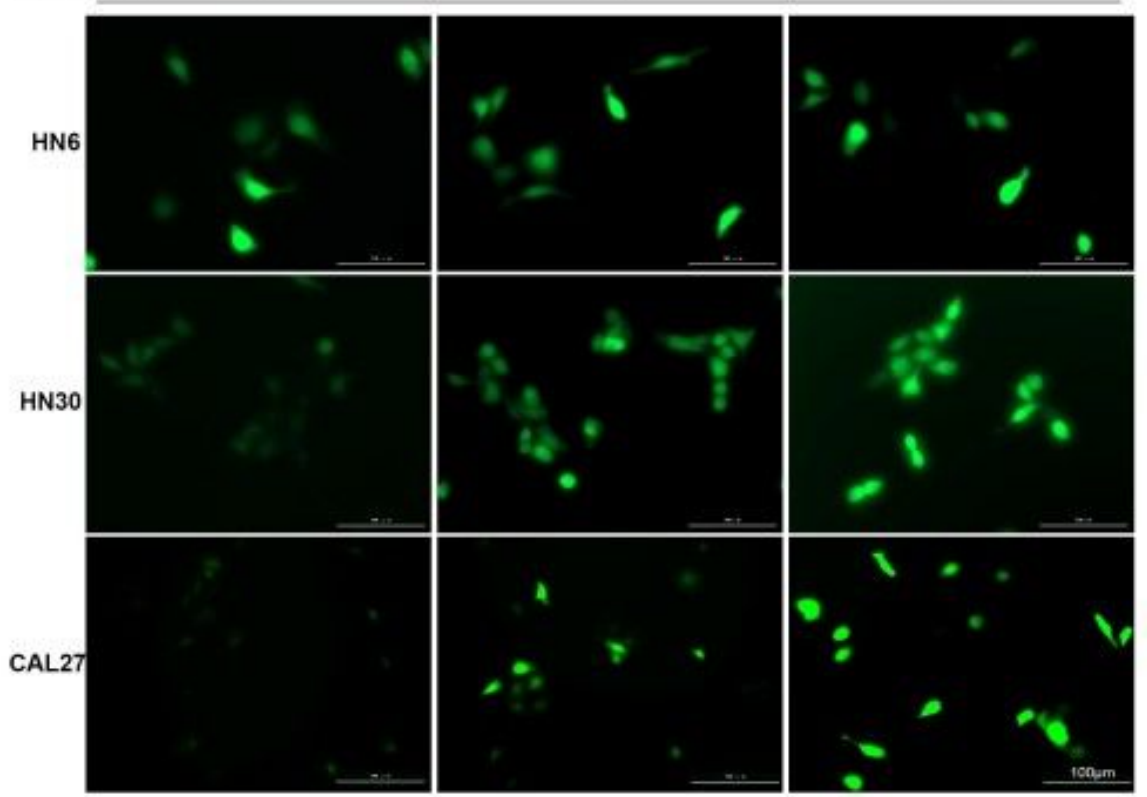

C

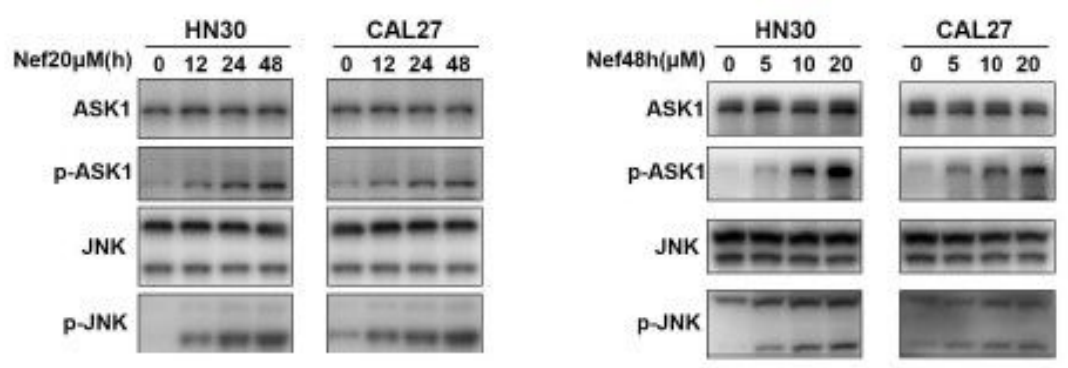

\section{Figure 3}

Neferine induced the ROS hypergeneration and activated the ASK1/JNK pathway to promote the apoptosis and autophagosome biosynthesis in HNSCC cells. (A) The flow cytometry analysis and histograms showed the amount of ROS in HNSCC cells treated with neferine for 24 hours using the oxidation-sensitive fluorescent probe dichlorofluorescein (DCF). (B) The fluorescence images showed the 
increase of ROS after neferine treatment. (C) Western blot of ASK1/JNK signal pathway in HNSCC cells treated with neferine in different conditons.

A
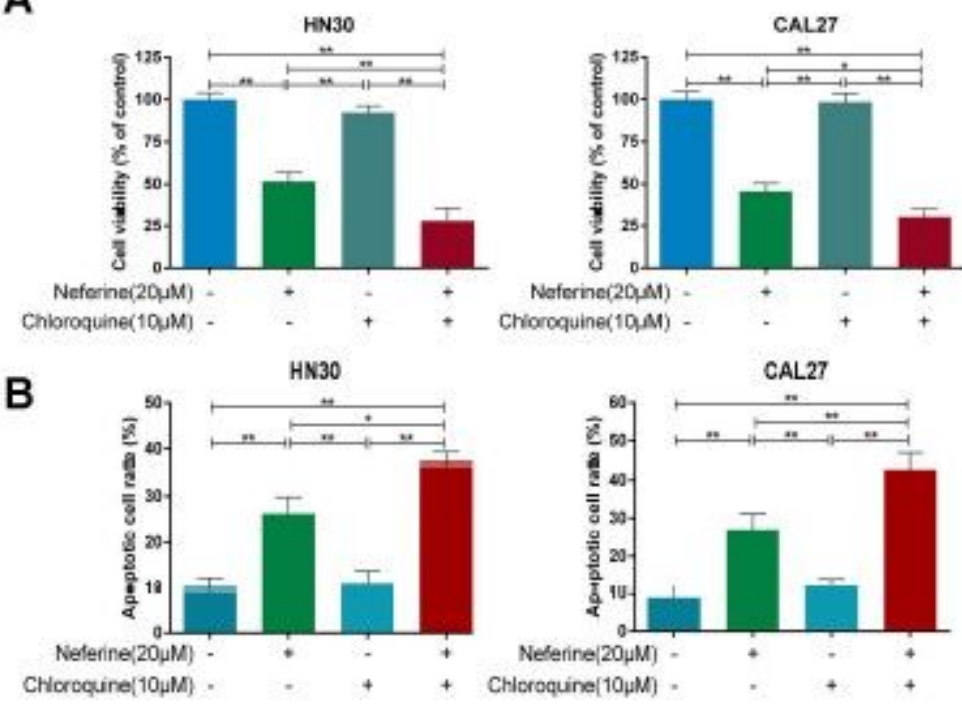

C

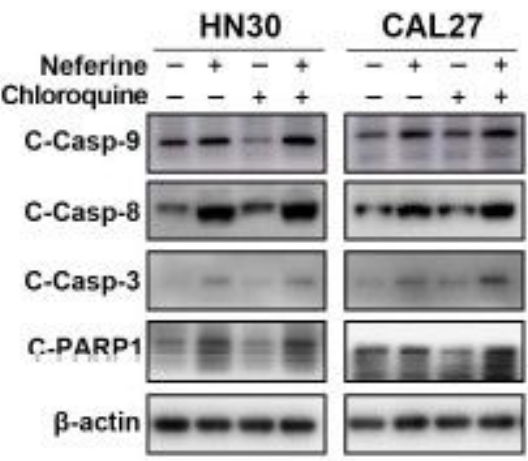

E

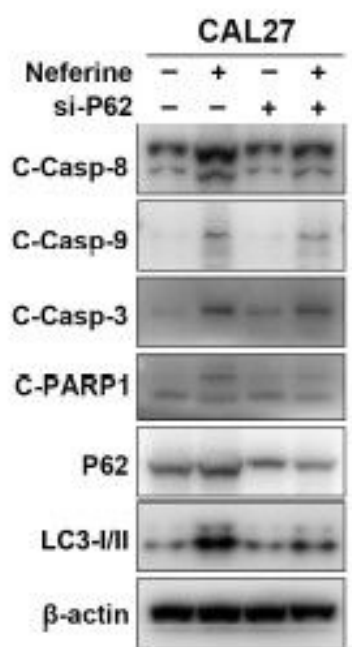

Figure 4

Neferine-induced apoptosis was partially mediated by the accumulation of p62 due to neferine-incuded autophagic influx inhibition in HNSCC cells. Cell viability (A), apoptosis cell rate (B) and western blot of cleaved caspase-8, caspase-9, caspase-3 and PARP1 (C) in the HN30 and CAL27 cells treated with neferine $(20 \mu \mathrm{M})$ in the presence or absence of chloroquine $(10 \mu \mathrm{M})$ for 48 hours. Flow cytometric analysis of early and late apoptosis (D) and western blot of cleaved caspase-8, caspase-9, caspase-3, PARP1, p62 and LC3 (E) in the neferine-treated HN30 and CAL37 cells with or without the knockdown of p62. 
A

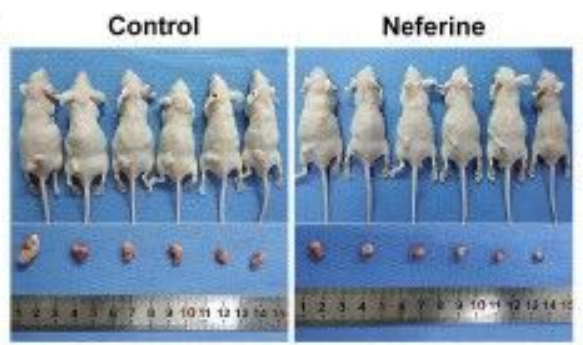

C
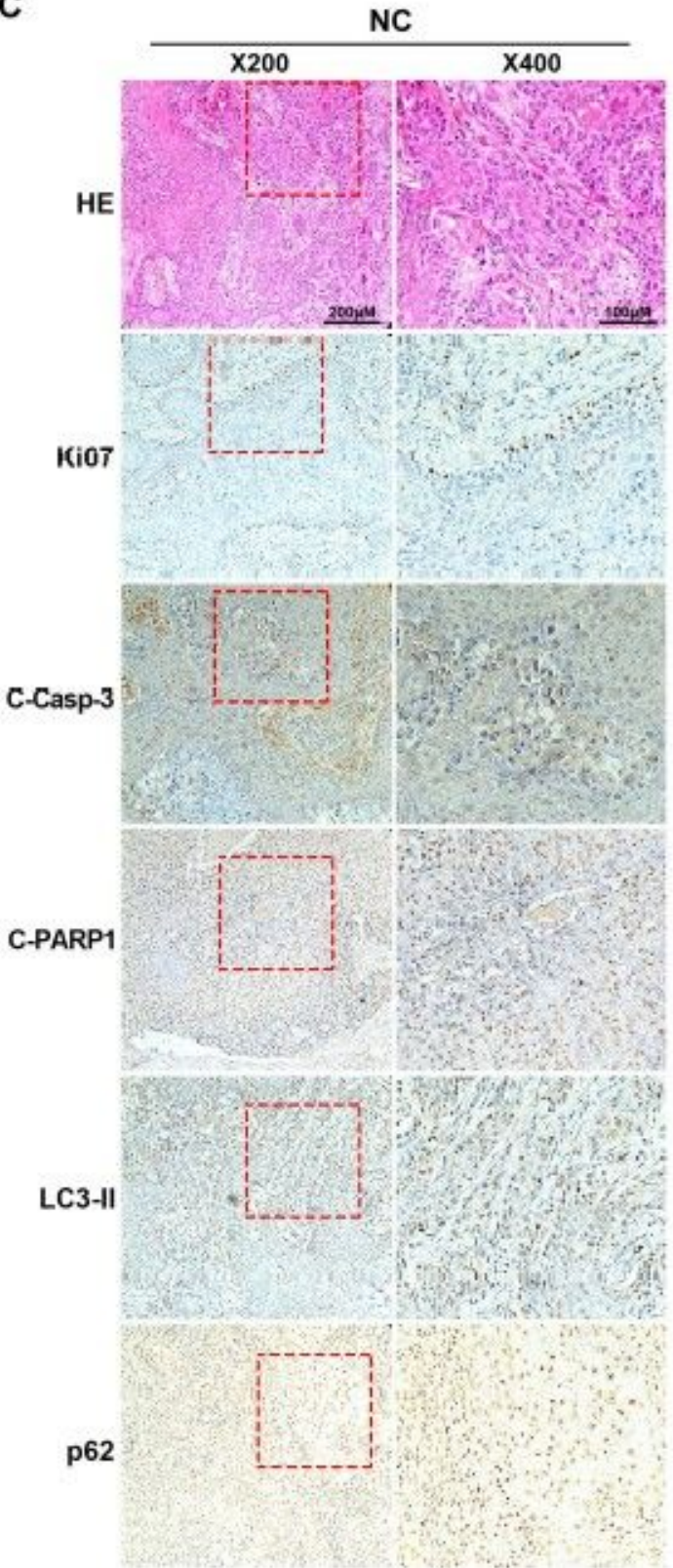

B
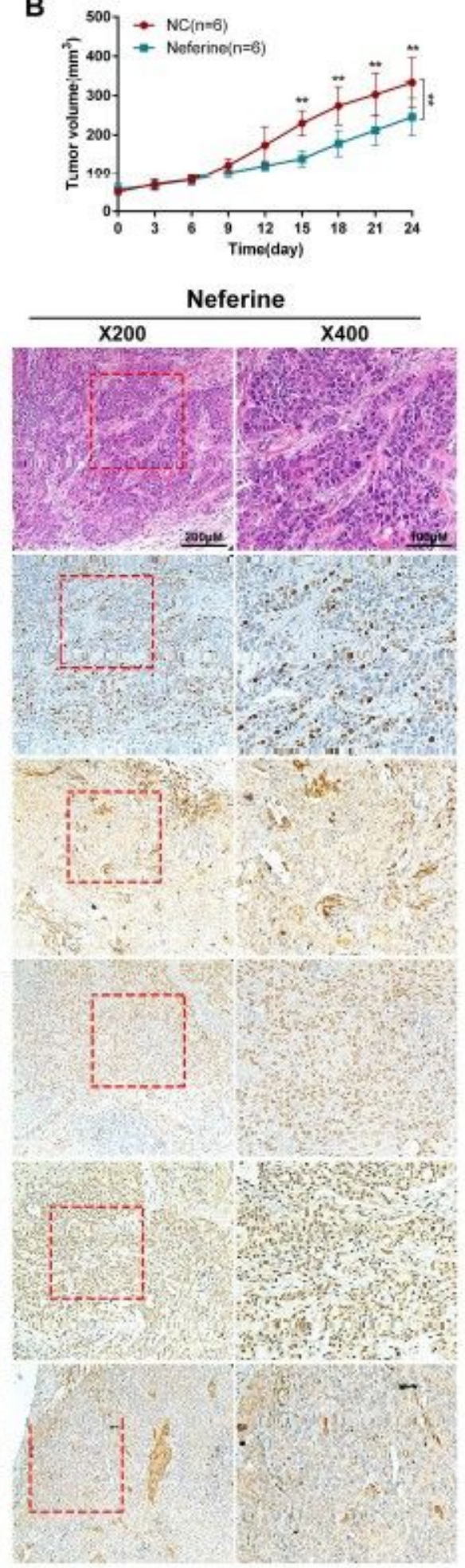

\section{Figure 5}

Neferine inhibited the HNSCC carcinoma growth by induction of apoptosis and autophagy in vivo. (A) Representative pictures of xenografts constructed by CAL27 cells, which were treated with vehicle or neferine. (B) The line chart showed growth rate of the tumors treated with neferine compared with the control. (C) The HE staining and Immunohistochemical staining of Ki67, cleaved caspase-3, cleaved PARP1, LC3 and p62 of xenograft tumor tissues treated with neferine or vehicle. 
A

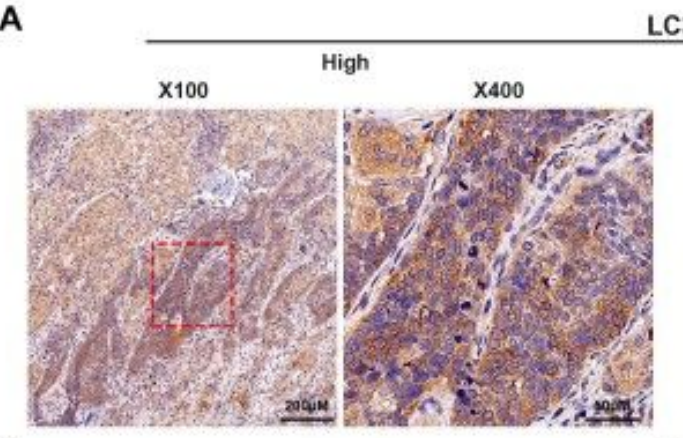

\section{LC3}

B
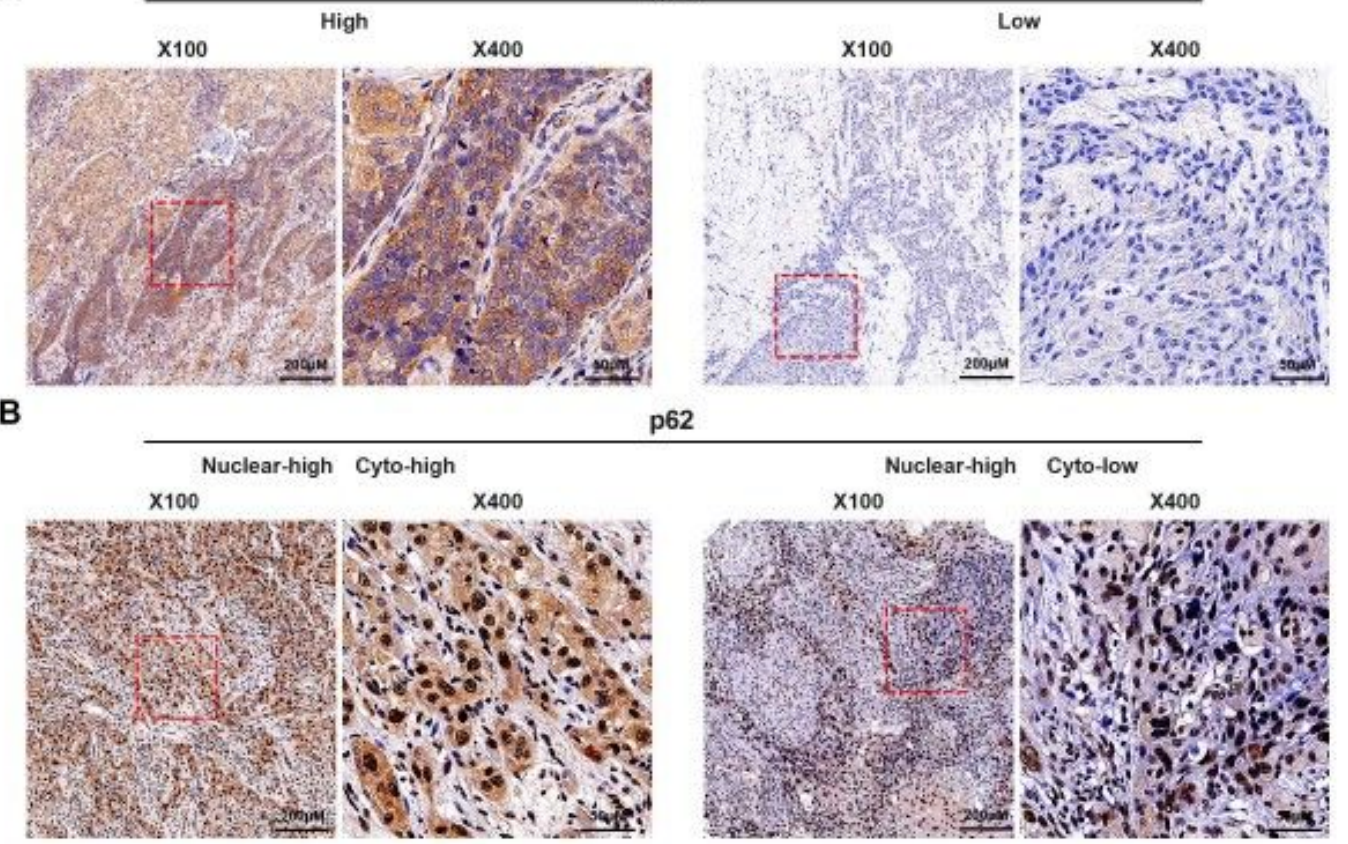

p62
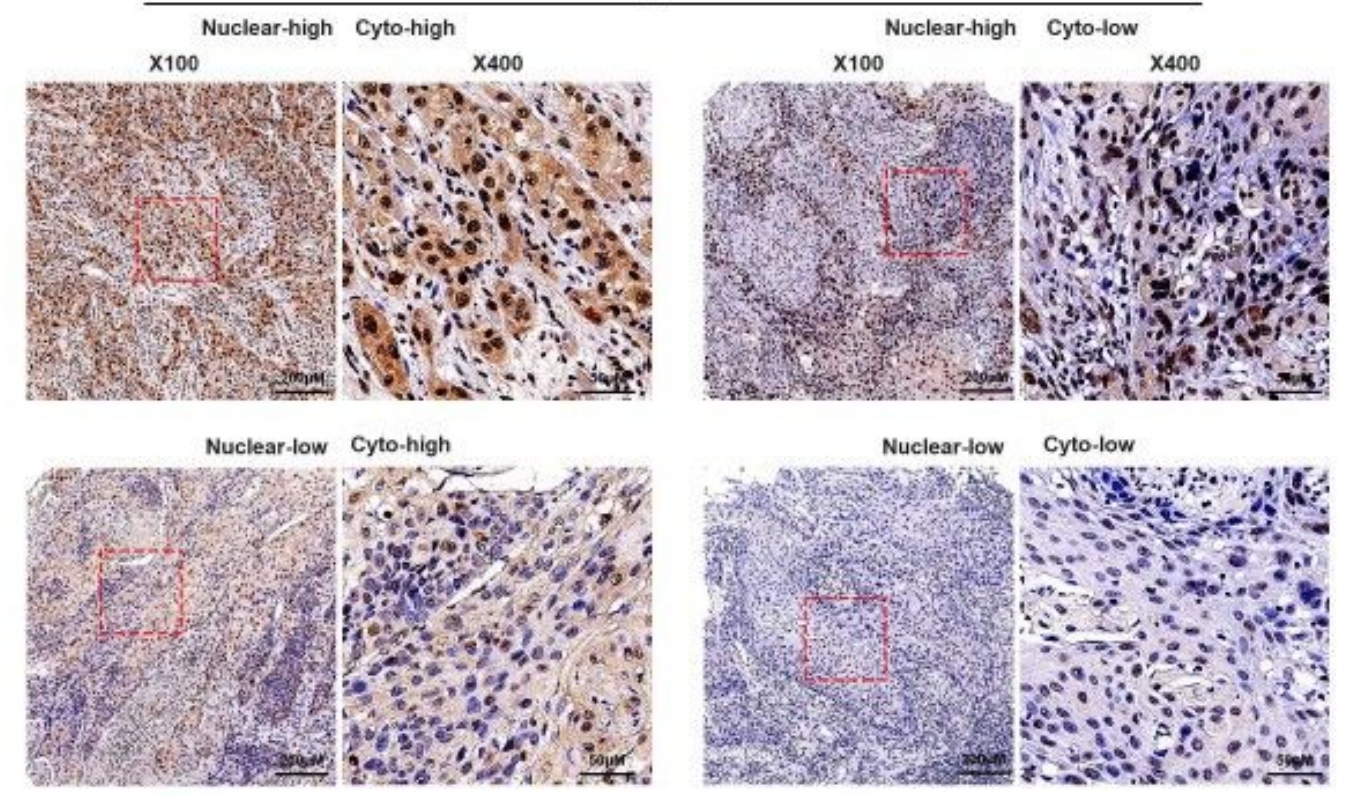

C
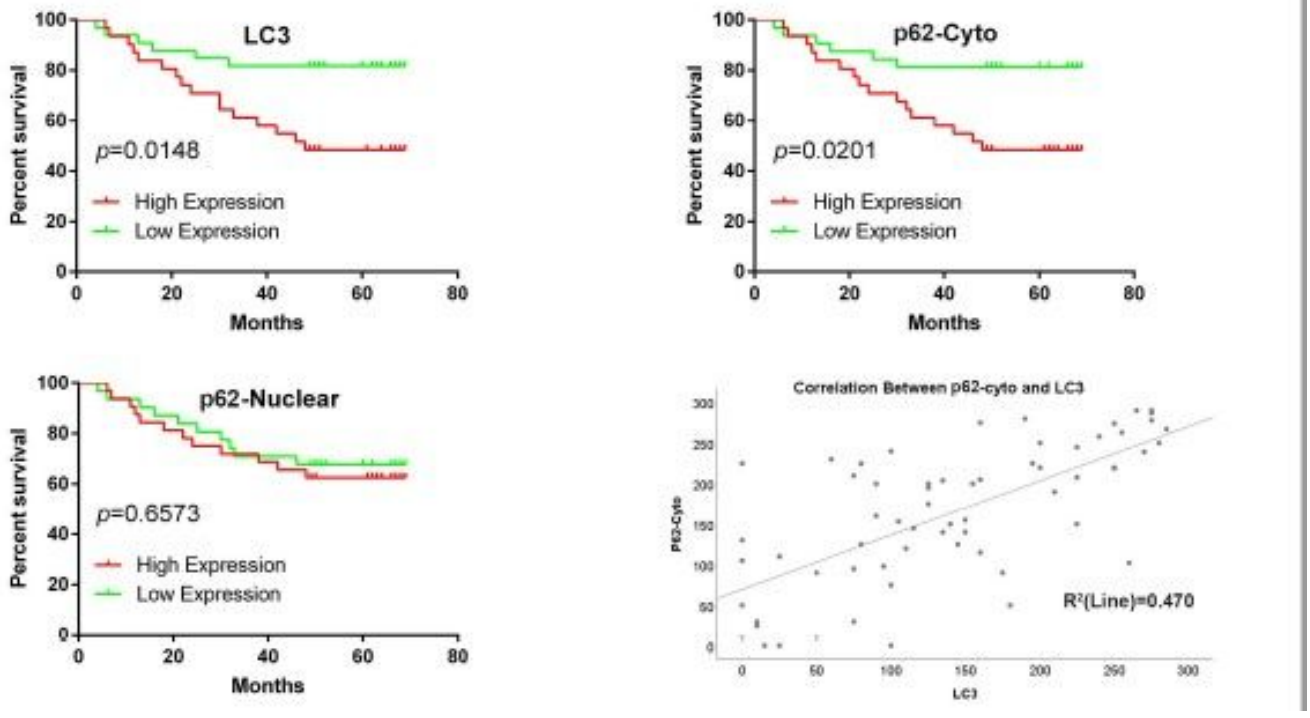

Figure 6

Pattern diagram of the pro-apoptosis by inhibiting autophagy flux mechanism exerted by Neferine.

\section{Supplementary Files}

This is a list of supplementary files associated with this preprint. Click to download. 
- supplementaryfigures13.pdf

- Supplementaryfigureslegends.docx

- Supplementaryfigureslegends.docx

- supplementaryfigures13.pdf 